\title{
Virtually Planned and Integrated Template-Guided Implant Surgical Placement: A Case Report
}

\author{
K.B.S. Kuckreja ${ }^{1,2}$ Harinder Kuckreja ${ }^{2}$ \\ ${ }^{1}$ The Tooth Place, Ludhiana, Punjab, India \\ 2Department of Prosthodontics, Baba Jaswant Singh Dental College, \\ Hospital and Research Institute Ludhiana, Ludhiana, Punjab, India
}

\begin{abstract}
Address for correspondence Harinder Kuckreja, MDS, Professor and Head, Department of Prosthodontics, Baba Jaswant Singh Dental College, Hospital and Research Institute Ludhiana, Ludhiana 141010 , Punjab, India (e-mail: hkuckreja@gmail.com).
\end{abstract}

Dent J Adv Stud 2018;6:40-42

\begin{abstract}
Keywords

- computer-aided design

- computer-assisted manufacture

- Smart Fusion

- surgical template

- guided implant surgery

The placement of dental implants in the maxillary anterior region is a challenge for clinicians because of existing anatomy and high esthetic demands. This article presents a case with limited anatomical three-dimensional space for implant placement and immediate loading for esthetic and functional restoration.

Virtual implant placement was planned using cone beam computed tomography (CBCT) scan, casts with trial denture bases, implant planning software (Nobel Clinician), and prosthetic designing software (Procera) to fabricate a surgical guide.

The article describes the successful application of "Smart Fusion" (Nobel Biocare) in the production of the surgical template for accurate placement of osseointegrated implants.
\end{abstract}

\section{Introduction}

In clinical oral implantology, cone beam computed tomography (CBCT) scan, computer-aided design, and computer-aided manufacturing (CAD/CAM) technology have proven to be invaluable in diagnosis, ${ }^{1}$ treatment planning, ${ }^{2}$ and surgical intervention. ${ }^{3}$ It enhances the clinician's ability to streamline the restorative outcome, communication with the patient, and reduce the surgical morbidity. The pretreatment workflow that precedes surgical implant placement is as important as or even more so than actual surgical intervention. Inadequate placement of oral implants may lead to esthetic or functional compromise in oral rehabilitation.

The surgical template or guide enables a predictable and a safe, minimally invasive surgery. Glossary of prosthodontic terms (GPT 8) defines surgical template as a guide used to assist in proper surgical placement and angulation of dental implants. The main objective of surgical guides fabricated with rapid prototyping and stereolithography (a laser-driven polymerization process that fabricates an anatomic model and surgical templates) is to translate the prosthetic implant treatment plan directly to the surgical field.

Fabrication of stereolithographic ${ }^{4,5}$ templates requires patient's computed tomography (CT) or CBCT image. Software packages are available that have been adapted to allow clinicians to virtually view the implant site and plan location, angle, depth, and diameter of virtual implants, which are superimposed on the three-dimensional (3D) data. Traditionally, following backward planning, the diagnostic wax up had to be visualized through CT scan with radiographic templates in place.

Several systems have been designed to fabricate surgical templates that transfer planned data from the computer to the recipient sites at the time of oral implant placement. Nobel Clinician (Nobel Biocare) is an interactive software program that allows for preoperative surgical planning. With CBCT data, optimal implant positions can be decided virtually and translated into a surgical template fabricated for surgery. Previous studies have shown that due to guided implantation, the accuracy is considerably improved compared with conventional template or freehand implant placement. ${ }^{6,7,8}$

\section{Case Presentation}

This clinical case report describes the treatment of a 17-year-old male patient with a congenitally missing maxillary left lateral incisor. Orthodontic treatment was done to create adequate space for the lateral incisor. Clinically, the space for lateral incisor seemed more than required. The patient was referred for restoration of missing left maxillary lateral incisor. ISSN 2321-1482.
License terms

(ㅇ) (1) $\ominus \circledast$ 
Intraoral periapical X-ray was taken, and diagnostic casts were prepared to assess the final prosthetic placement. Patient was advised for implant-supported single tooth replacement; hence, CBCT was recommended. Nobel Clinician software (Nobel Biocare) was used to relate parameters for the final implant placement. It was observed that the teeth numbers 21 and 23 had their roots tilted into the edentulous space (-Fig. 1), whereby making the implant space very narrow and precariously close to the apical third of roots of adjacent teeth.

It was decided to insert a narrow platform (NP) $3.5 \mathrm{~mm}$ NobelActive Tapered Implant with Nobel Surgical Guide fabricated through a technique called, "Smart Fusion."

CBCT was done using Kodak Carestream 6300 scan, and the Digital Imaging and Communications in Medicine (DICOM) data were simulated using Nobel Clinician software.

Maxillary teeth were prepared by blocking out the orthodontic appliances with gingival mask, and a diagnostic cast was prepared by making a maxillary impression with Addition Silicon rubber-based impression material (Virtual Putty and Low viscosity material, Ivoclar Vivadent). The trial denture base for missing left maxillary lateral incisor was made with prosthetic teeth arrangement to recreate form and function and esthetics ( - Fig. 2). The try in was completed to verify the esthetic and functional requirements of the prosthesis.

The cast and the cast with the trial denture base were scanned with Procera Laboratory $2 \mathrm{G}$ scanner, and the 3D scan data were transferred to the Nobel Clinician software.

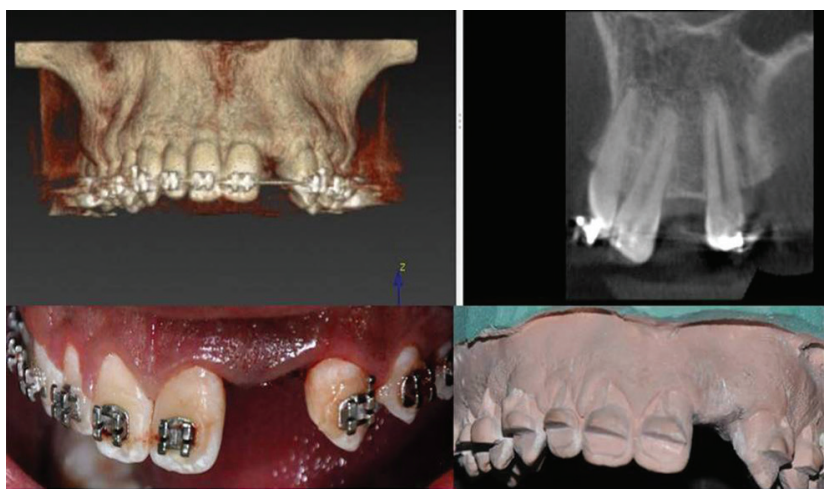

Fig. 1 Preoperative situation of missing tooth number 22, СВCT simulation, and cast of the partially edentulous arch. CBCT, cone beam computed tomography.

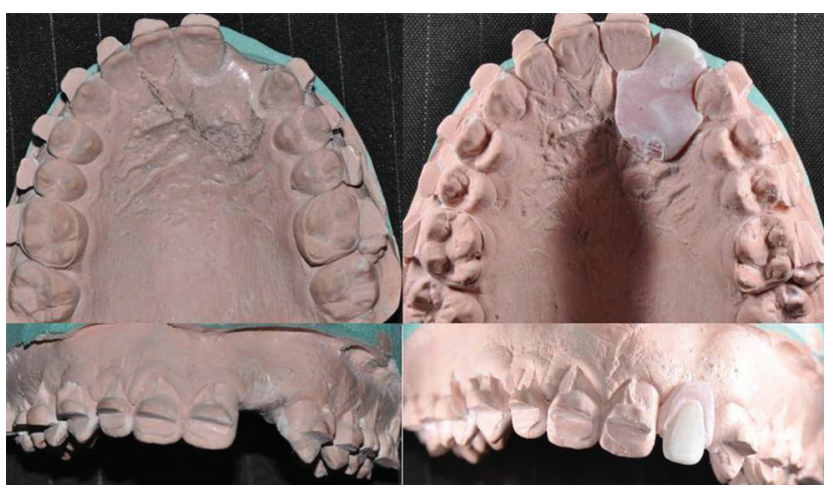

Fig. 2 Cast and trial denture base of tooth number 22 ready for scanning.
The Nobel Clinician software merges the CBCT data files of the patient with the cast and the prostheses to give us a composite data of all the scans for virtual planning of implant location, angulations, and emergence according to the correct prosthetic location of the final prosthesis-"Smart Fusion" (-Fig. 3).

The correct selection of implant from the implant library in the software was done. NobelActive NP $3.5 \mathrm{~mm}$ and 18 $\mathrm{mm}$ long implant was selected, and correct 3D positioning was verified and saved.

The software generates the surgical guide ( - Fig. 4) for the same implant with accurate positioning parameters from data of cast, adjacent teeth, and CBCT. The anchor pins to stabilize the guide are also planned for stabilization during the surgical drilling protocol.

The whole planning is checked for accuracy of planning, implant selection, angulations, and position of anticipated abutment and crown. The order is generated through the software, and the surgical guide is prepared with stereolithography and 3D printing.

The surgical guide was received in a sterile state and checked in patients' mouth during surgical visit after following all the surgical and disinfection protocols.

The patient was prepared for the surgical implant placement. Guided surgical kit for implants is required for implant osteotomy and insertion (-Fig. 5).

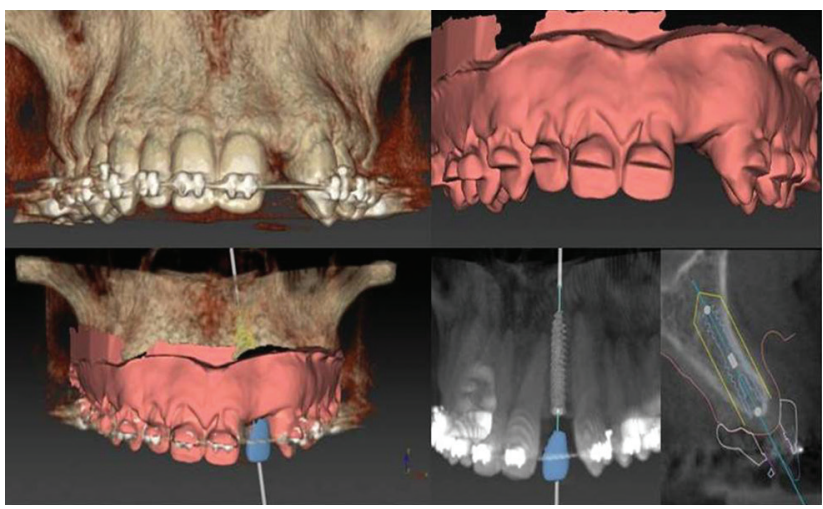

Fig. 3 Nobel clinician simulation of situation, scanned image of the cast, and trial denture base fused for implant simulation-Smart Fusion.

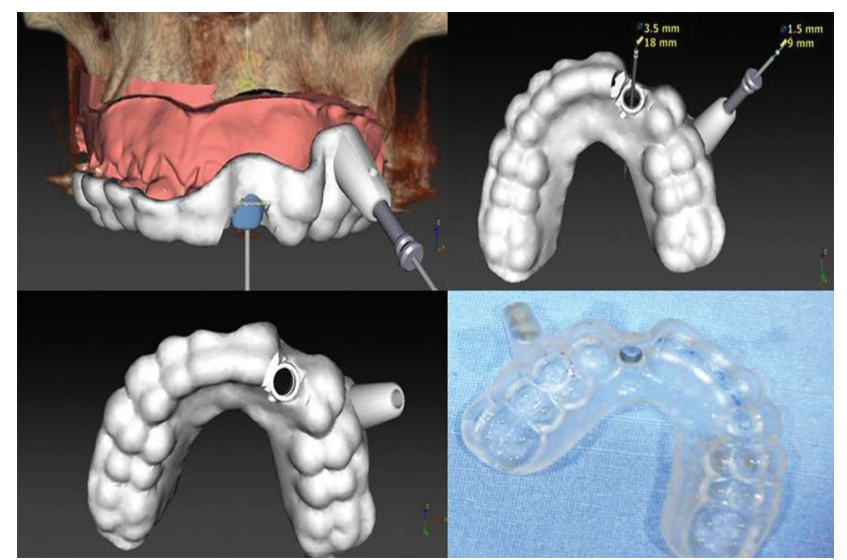

Fig. 4 Software-generated surgical guide template and guide template after stereolithographic fabrication. 

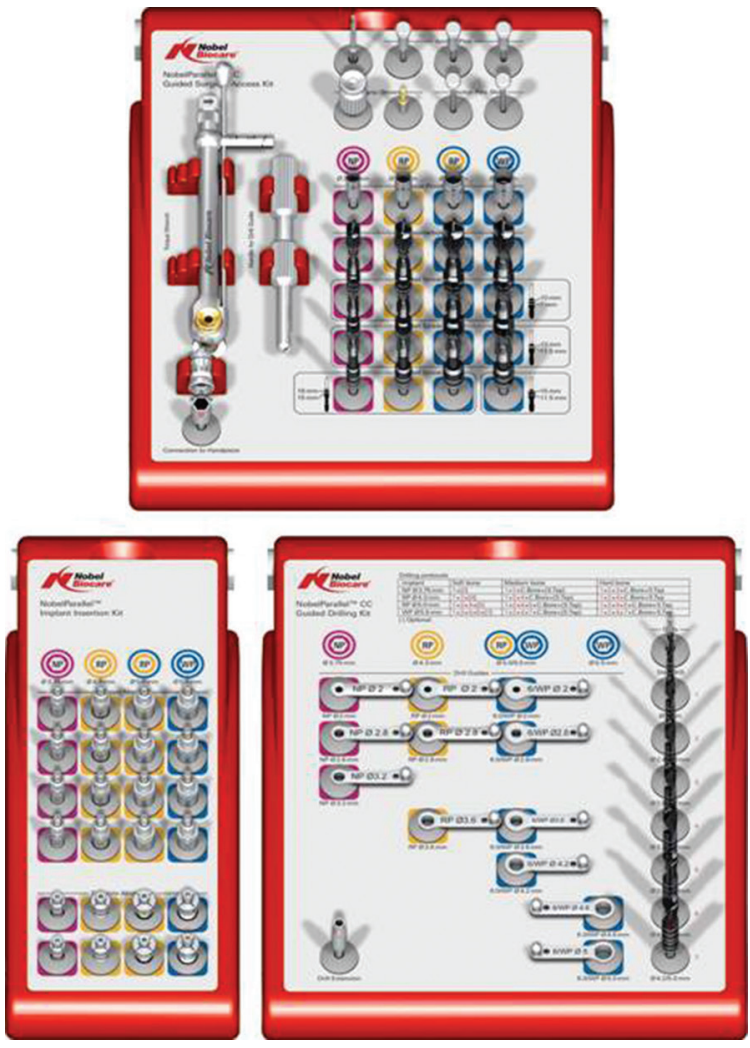

Fig. 5 Guided implant surgical kit (Nobel Biocare).

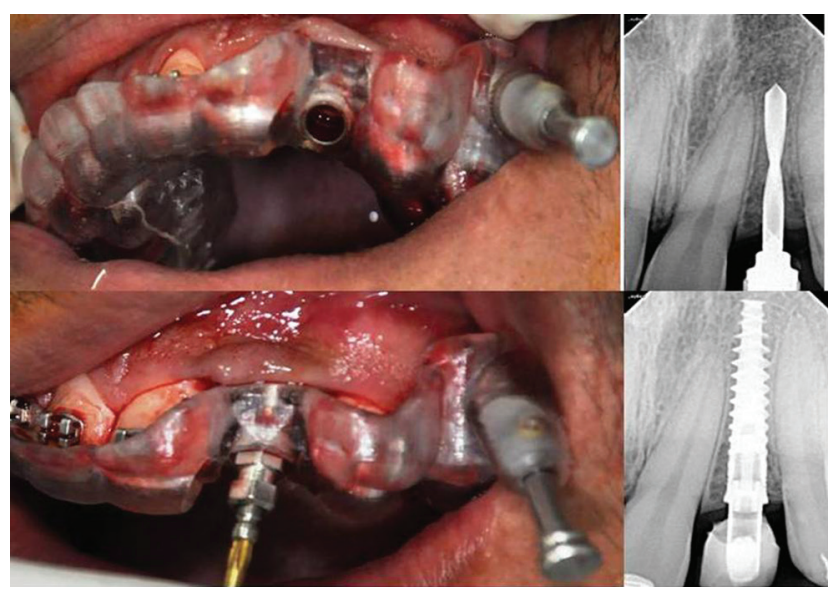

Fig. 6 Surgical guide template is secured in the mouth and implant insertion done.

The surgical guide is retained and secured with the guide pins at the planned strategic locations ( - Fig. 6). The surgical protocol is planned for tissue punch, initial pilot drill, osteotomy with successive drills, and final screw tap for creating implant seat. Intraoral X-ray view is done at pilot drill stage to verify the corono-apical and mesio-distal position of the intended implant location.

The implant is inserted with implant driver and surgical hand piece with maximum torque limited at $35 \mathrm{Ncm}$. As soon as the implant is inserted, another $\mathrm{X}$-ray view is done to confirm the final implant position.

Impression with open tray impression material (addition silicon putty) is made and sent to laboratory for fabrication

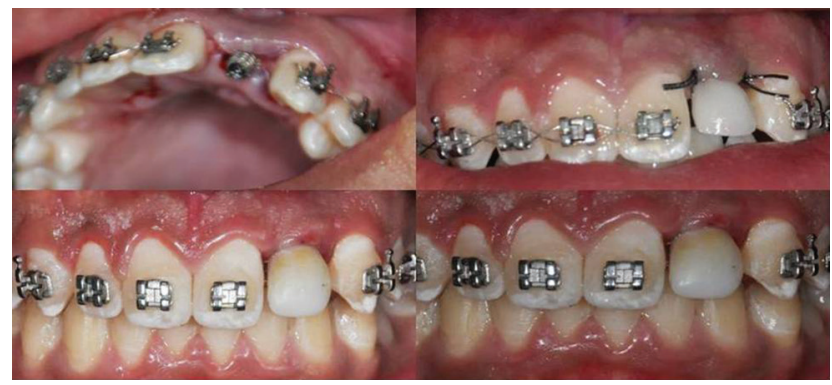

Fig. 7 Temporary titanium cylinder and composite screw retained temporary restoration given immediately.

of immediate composite crown with temporary titanium cylinder ( - Fig. 7).

Screw-retained composite temporary crown is attached in the mouth. Follow-up after 1 week, 1 month, and 3 months is planned for routine check-up and fabrication of the final restoration

Final Prostheses is planned as screw retained zirconia implant crown with ASC (Angulated screw access)

\section{Conclusions}

The precision of clinical treatment provided by the integration of CAD/CAM and CBCT allows dentists to plan for ideal surgical placement and the appropriate selection of restorative modalities before placing implants.

\section{Conflict of Interest}

None.

\section{References}

1 Tahmaseb A, Wismeijer D, Coucke W, Derksen W. Computer technology applications in surgical implant dentistry: a systematic review. Int J Oral Maxillofac Implants 2014;29(Suppl):25-42

2 Vercruyssen M, Laleman I, Jacobs R, Quirynen M. Computersupported implant planning and guided surgery: a narrative review. Clin Oral Implants Res 2015;26(Suppl 11):69-76

3 Widmann G, Fischer B, Berggren JP, et al. Cone Beam computed tomography vs multislice computed tomography in computer aided design/computer assisted manufacture guided implant surgery based on three dimensional optical scanning and stereolithographic guides: does image modality matter? Int J Oral Maxillofac Implants 2016;31(3):527-533

4 Dandekeri SS, Sowmya MK, Bhandary S. Stereolithographic surgical template: a review. J Clin Diagn Res 2013; 7(9):2093-2095

5 Cassetta M, Di Carlo S, Pranno N, Sorrentino V, Di Giorgio G, Pompa G. The use of stereolithographic surgical templates in oral implantology. Ann Ital Chir 2013;84(5):589-593

6 Sarment DP, Sukovic P, Clinthorne N. Accuracy of implant placement with a stereolithographic surgical guide. Int J Oral Maxillofac Implants 2003;18(4):571-577

7 D’haese J, Van De Velde T, Komiyama A, Hultin M, De Bruyn $\mathrm{H}$. Accuracy and complications using computer-designed stereolithographic surgical guides for oral rehabilitation by means of dental implants: a review of the literature. Clin Implant Dent Relat Res 2012;14(3):321-335

8 Stübinger S, Buitrago-Tellez C, Cantelmi G. Deviations between placed and planned implant positions: an accuracy pilot study of skeletally supported stereolithographic surgical templates. Clin Implant Dent Relat Res 2014;16(4):540-551 